\title{
Phasors Estimation at Off-Nominal Frequencies through enhanced-SVA method with Fixed Sampling Clock
}

\author{
Christophe Ghafari, Hamada Almasalma, Bertrand Raison, Member, IEEE, Nouredine Hadjsaid, \\ Senior Member, IEEE, Raphaël Caire, Senior Member, IEEE, Eric Martin
}

\begin{abstract}
This paper provides a solution to estimate synchrophasors with high accuracy at off-nominal frequencies using a nominal frequency clock. The sampling rate is fixed, while the amplitude of the sampled values is altered to generate a sequence of samples whose sampling frequency is synchronized to the power system frequency. The re-computation of the synchronized samples is done by cubic spline interpolation. The algorithm requires knowledge of the fundamental frequency of the power system which is estimated based on the phase angle difference between two phasors. The performance of the algorithm has been tested by simulation, through different scenarios, following the test conditions described in the IEEE Standard C37.118.1-2014a. The test cases include: steady-state with off-nominal frequencies, harmonic distortions, amplitude and phase modulations, positive and negative ramps of frequency, and frequency jump. The simulation results show the technique's capability in efficiently dealing with harmonics and off-nominal frequencies under static and dynamic conditions.
\end{abstract}

Index Terms--Asynchronous sampling, frequency measurement, phasor, phasor measurement unit (PMU), protection relay, Discrete Fourier transform (DFT), sample value adjustment (SVA), synchrophasor measurement.

\section{INTRODUCTION}

$\mathrm{P}$ OSITIVE sequence voltage and current phasor estimates are the most important parameters in several monitoring, control and protection functions in power system [1]. When these phasors are separated by hundreds of miles, it becomes necessary to synchronize the measurement process (synchrophasor), to ensure an effective way to monitor the real-time operation status of a wide-area measurement system (WAMS) improving substantially their reliability, observability and security [2].

Fortunately, advances in electronic engineering and high speed communications have made precise time synchroniza-

C. Ghafari is with the Grenoble Electrical Engineering laboratory (G2Elab), Grenoble 38031, France and the Schneider Electric SAS, Meylan 38240, France (e-mail: christophe.ghafari@schneider-electric.com).

H. Almasalma is with the Department of Electrical Energy and Computer Architectures, Katholieke Universiteit Leuven (KU Leuven), Leuven 3000, Belgium (e-mail: hamada.almasalma@kuleuven.be).

N. Hadjsaid, R. Caire, and B. Raison are with the Grenoble Electrical Engineering laboratory (G2Elab), Grenoble 38031, France (e-mail: nouredine.hadjsaid@g2elab.grenoble-inp.fr; raphael.caire@g2elab.grenobleinp.fr; bertrand.raison@g2elab.grenoble-inp.fr).

E. Martin is with the Schneider Electric SAS, Meylan 38240, France (email: eric-pierre.martin@schneider-electric.com). tion in the power grid more practical and cost-effective. The emergence of Global Positioning System (GPS) technology brought the solution to the power system wide area measurement. It is shown that the GPS offers the most effective way of synchronizing power system measurements over relatively long distances. Through the use of GPS, time synchronization less than 1 microsecond is now realized [3].

Phasors are typically used for protective relays and phasor measurement units (PMUs) [4], [5]. The Discrete Fourier Transform (DFT) is the most common method used for phasor measurements as it provides simple, elegant and accurate estimation of phasors [6]. There are number of alternative non-DFT-type algorithms for estimating the phasors under power system frequency variations as Weighted Least Squares (WLS) [7] and Kalman filter [8]. But these algorithms are complex to implement and require higher computational effort than the DFT estimate.

The frequency of the Analog-to-Digital Converter (ADC) should be fixed and synchronized to the nominal frequency in order to obtain synchronized phasor measurements [9]. As frequency is constantly changing in power systems due to changes in load and generation imbalances, especially with the wide use of renewable energy sources and distributed generation [10], the fixed sampling frequency cannot be always synchronized to the power system frequency.

When the fixed sampling frequency is not synchronized with the power system frequency, the DFT is not able to correctly estimate the phasor. This is due to the fact that the DFT window will contain non-integer number of cycles. In the DFT theory, the input signal is assumed to be periodic. For a sinusoidal signal, when there is an integer number of cycles within the data window, the assumed periodic waveform is the same as an infinite duration pure sinusoid. However, if the data window contains a non-integer number of cycles of a sinusoid then the assumed periodic waveform will not be a pure cosine wave and will have end-point discontinuities which make the DFT wrongly estimate the phasors. This pernicious phenomenon is well known as leakage effect [11].

\section{OVERVIEW OF THE POSSIBLE SOLUTIONS}

There are five main families of solutions to solve the leakage problem and give back the DFT its inherent robustness to estimate the parameters of interest. The first solution is 
the use of the frequency tracking [12]. The basic idea is to use the estimated frequency to synchronize the sampling frequency of the ADC clock to the power system frequency. However, the resulting phasor measurements are not referenced to absolute time, so that synchronized phasor measurement applications to an entire power system are not possible.

The second solution consists in using an algorithm after the DFT algorithm to correct the calculated phasor based on the estimation of the actual frequency [13], [14]. The drawbacks of this solution are that the correction is only done for the fundamental phasor and there is no adjustment for the phasors of the harmonics. A high order filter is required to remove the harmonics as the derivation of the algorithms does not include the harmonics effect. The use of filters introduces delays and increases the computational burden.

The third solution consists in using a variable DFT window length to reduce the leakage error by synchronizing the DFT window to the estimated frequency [15], [16]. However, the procedure is applicable only for discrete fundamental frequencies. An error will occur in the phasors estimate obtained through the DFT for other fundamental frequencies. Also this would increase the computational burden.

The fourth solution consists in exploiting the combination between windowing functions [17] with the interpolateddiscrete Fourier transform (IpDFT), first introduced in [18]. In this approach, both long-range and short-range leakages can be reduced, as presented in [19], where the Hanning window has been selected to show the superiority of the IpDFT with tapering over the classical IpDFT algorithm. Consequently, this solution has been applied to estimate synchrophasors and the performance of the algorithm have been evaluated through the Total Vector Error (TVE) criterion in both steady-state and dynamic conditions following the IEEE Standard C37.118.12011 [20] for the M-Class PMU in [21]. Subsequently, a lot of investigations have been realized for better characterization of the tapered IpDFT in terms of windowing functions [22], size of the observation interval [23], and sampling rate [24] for both performance classes; P-Class and M-Class PMU. Furthermore, [24] has proposed an enhanced-IpDFT (e-IpDFT) to compensate for the spectral interference introduced by the negative image of the DFT spectrum and has shown very promising performances through real-time implementation. To conclude, most of these references solve the leakage problem of the DFT for off-nominal frequencies under both steadystate and dynamic conditions, making IpDFT algorithms very promising techniques. However, the aforementioned Ip-DFT algorithms, based on Hanning window, require a sampling rate much higher than the fundamental frequency (typically some tens of $\mathrm{kHz}$ ) to avoid aliasing effect and make possible specific approximations needed by the algorithm [25]. Additionally, a minimum window length of 2 cycles is required to ensure that the distance between spectral lines suffices to allow effective leakage reduction by windowing [26].

The fifth solution is the resampling techniques. These techniques aim at modifying the input samples towards an ideal signal sample sequence, whose sampling frequency is synchronized to the power system frequency. Phasor estimation is then performed using the resampled data. Reference [27] shows that resampled data phasors have very little errors of estimation. The resampling technique cited above is based on trigonometric function which does not include the effect of the harmonics, that is why a high order filter is needed to send a pure sinusoidal signal to the algorithm. If the phasors of the harmonics are required, then a bank of FIR (Finite Impulse Response) filters is needed. The use of high order filter makes the system response slower.

The objective of this paper is to derive a resampling equation that does not depend on the shape of the signal and can give high accuracy of synchronization between the sampling frequency and the power system frequency.

The rest of the paper is organized as follows. Section III gives the theoretical background in case of asynchronous sampling. Section IV develops a solution to the DFT leakage problem based on resampling technique. The performance of the proposed algorithm is analyzed in Section V. Finally, conclusions of the paper are summarized in Section VI.

\section{THEORETICAL BACKGROUND OF ASYNCHRONOUS SAMPLING}

The following condition should be satisfied in order to ensure synchronization between the sampling frequency and the power system frequency

$$
f_{S}=N \times f
$$

where $f_{S}$ is the synchronous sampling frequency, $N$ is the number of samples per cycle and $f$ is the power system frequency. Now consider a sinusoidal signal $x(t)$ of frequency $f$ given by (2)

$$
x(t)=A \cdot \cos (2 \pi f t+\phi)
$$

where $A$ and $\phi$ are the amplitude and phase angle of the fundamental component of $x(t)$ respectively. If the signal $x(t)$ is sampled based on the sampling frequency $f_{S}$ as in (1), then the samples set $x[n]$ can be expressed as (3)

$$
x[n]=A \cdot \cos \left[2 \pi \frac{n}{N}+\phi\right]
$$

where $N$ samples are acquired in exactly one signal cycle.

For synchrophasor estimation, the sampling frequency should be fixed and synchronized to the nominal frequency $f_{0}$ $(50 \mathrm{~Hz}$ or $60 \mathrm{~Hz})$ as shown in (4)

$$
f_{s 0}=N \times f_{0}
$$

where $f_{s 0}$ is the asynchronous sampling frequency. If the signal $x(t)$ is sampled based on $f_{s 0}$, then the samples set is given by (5), which is different from the synchronous samples of (3)

$$
x[n]=A \cdot \cos \left[2 \pi f \frac{n}{N f_{o}}+\phi\right]
$$


where $x[n]^{\top}$ refers to asynchronous samples set. It can be shown that the fundamental phasor computed by the nonrecursive DFT updates based on the asynchronous samples set $x[n]$ " can be given by (6).

$$
X^{\prime}{ }_{r}=P \cdot X_{0} \cdot e^{j r \omega \Delta t_{o}}+Q \cdot X_{0}^{*} \cdot e^{-j r \omega \Delta t_{o}}
$$

where $X^{\prime}{ }_{r}$ is the wrong estimated phasor due to the asynchronous sampling, $X_{0}$ is the correct phasor, * denotes complex conjugate, $\omega$ is the off-nominal angular frequency $(\omega=2 \pi \mathrm{f}), \Delta t_{o}$ is the asynchronous sampling period, and $P, Q$ are defined as

$$
\begin{array}{r}
P=\frac{\sin \left(\frac{N\left(\omega-\omega_{o}\right) \Delta t_{o}}{2}\right)}{N \sin \left(\frac{\left(\omega-\omega_{o}\right) \Delta t_{o}}{2}\right)} e^{j(N-1)\left(\frac{\omega-\omega_{o}}{2}\right) \Delta t_{o}} \\
Q=\frac{\sin \left(\frac{N\left(\omega+\omega_{o}\right) \Delta t_{o}}{2}\right)}{N \sin \left(\frac{\left(\omega+\omega_{o}\right) \Delta t_{o}}{2}\right)} e^{-j(N-1)\left(\frac{\omega+\omega_{o}}{2}\right) \Delta t_{o}}
\end{array}
$$

where $\omega_{0}$ is the nominal angular frequency $\left(\omega_{0}=2 \pi f_{0}\right)$.

The detail derivation of (6) can be found in [28].

\section{DESCRIPTION OF THE PROPOSED SOLUTION}

This section presents the algorithm of resampling that aims at adjusting the value of the samples (amplitude correction) using spline interpolation in order to generate a sequence of samples whose sampling frequency is matched to the power system frequency to ensure that there are no errors in the synchrophasor estimates based on DFT techniques.

\section{A. Basic Idea}

The idea of the resampling based on amplitude correction is to alter the magnitude of the samples. The re-computed samples will have the same magnitude as if the signal was sampled based on sampling frequency that is matched to the off-nominal frequency. As shown in Fig. 1, the goal is to alter the magnitude of asynchronous samples generated from sampling the signal $x(t)=\cos (2 \pi(55) t+\pi / 4)$ at asynchronous sampling frequency $1200 \mathrm{~Hz}\left(N \times f_{o}=24 \times 50\right)$ to be as the magnitude of the synchronous samples generated from sampling the same signal at synchronous sampling frequency $1320 \mathrm{~Hz}(N \times f=24 \times 55)$. This technique acts as the sampling period has been changed so that the sampling frequency matches the power system frequency.

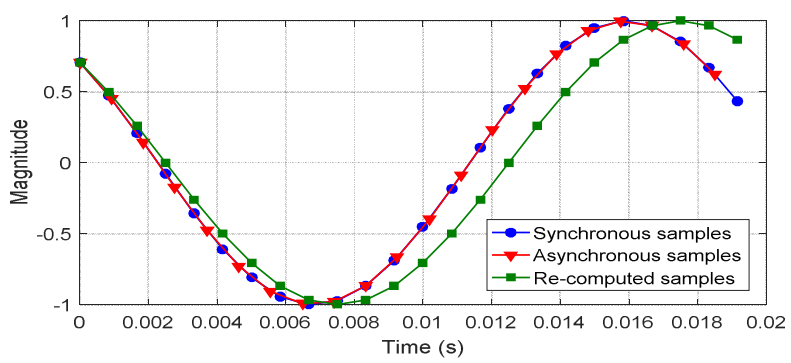

Fig. 1. Basic idea of the resampling algorithm.

\section{B. Derivation of the Algorithm}

The basic idea of the algorithm is to convert the asynchronous samples set $x[n]^{\boldsymbol{\top}}$ to the synchronous samples set $x[n]$ by altering the magnitude of the samples. To do so, an equation has to be derived that expresses $x[n]$ as function of $x[n]$. Let us suppose that the time drift between the asynchronous sampling period $\Delta t_{o}$ and the synchronous sampling period $\Delta t_{s}$ is given by (9).

$$
\Delta t_{0}-\Delta t_{s}=\frac{1}{f_{s 0}}-\frac{1}{f_{s}}=e
$$

$x[n]$ and $x[n]$ "an be rewritten as

$$
\begin{gathered}
x[n]=x\left[n \Delta t_{s}\right]=A \cdot \cos \left[2 \pi f n \Delta t_{s}+\phi\right] \\
x[n]=x\left[n \Delta t_{o}\right]=A \cdot \cos \left[2 \pi f n \Delta t_{o}+\phi\right]
\end{gathered}
$$

Now based on (9), $x\left[n \Delta t_{s}\right]$ can be expressed as

$$
x\left[n \Delta t_{s}\right]=x\left[n \Delta t_{o}-n e\right]
$$

Reference [29] uses the first order Taylor Series around the point $n \Delta t_{o}$ to find an equation that gives $x[n]$ as function of $x[n]$ ". 2 cycles of samples are needed in order to correct one cycle of samples, which introduces delays. Also the algorithm does not give high accuracy but the idea is interesting.

Reference [30] has modified the concept by extending the Taylor series till the second derivative. Reference [30] claims that its proposed algorithm gives a maximum magnitude error of less than $0.1 \%$ for frequency deviations up to $10 \%$ from nominal. The claim is valid only if the signal does not contain harmonics higher than the second order, i.e. third harmonic. But in case of higher order harmonics, the magnitude error will be near $1 \%$ for steady state conditions. Also, [30] has not specified the frequency estimation algorithm necessary to run its resampling algorithm. This might be an issue as frequency measurements affect the accuracy of the resampling algorithm and the computational burden. Finally, the results claimed by [30] are valid only for IEEE Standard C37.118-2005 [31], which does not cope with dynamic performances of synchrophasors, and has been superseded successively by [20] and IEEE Standard C37.118.1a-2014 [32] (Amendment to IEEE Standard C37.118.1-2011 on performances requirements).

This paper has modified the method of [30] by using the third order Taylor series that is to have more accuracy over wider range of off-nominal frequencies. Also, this paper has integrated the modified algorithm with the Fourier frequency estimation algorithm that is based on the phase angle difference between two phasors. The frequency estimation based on Fourier algorithm is very simple to implement and the additional amount of computational burden is minimal. Lastly, our algorithm has been designed to deal with dynamic performances introduced by the latest version of the IEEE standard.

Using Taylor Series till the third derivative around the point $n \Delta t_{o}$, (12) can be written as 


$$
\begin{aligned}
x[n]=x\left[n \Delta t_{o}-\right. & n e] \\
& =x\left[n \Delta t_{o}\right]-(n e) \cdot x^{\prime}\left[n \Delta t_{o}\right] \\
& +\frac{1}{2}(n e)^{2} \cdot x^{\prime \prime}\left[n \Delta t_{o}\right] \\
& -\frac{1}{6}(n e)^{3} \cdot x^{\prime \prime \prime}\left[n \Delta t_{o}\right]
\end{aligned}
$$

(ne) can be written as $(\alpha h)$, where $\alpha=n \Delta f=n\left(\frac{f-f_{o}}{f}\right)$ and $h=\Delta t_{o}$. Based on this, (13) can be written as

$$
\begin{aligned}
x[n]=x[n] & -(\alpha h) \cdot x^{\prime}[n] \mathbf{\bullet}^{\mathbf{}} \\
& +\frac{1}{2}(\alpha h)^{2} \cdot x^{\prime \prime}[n] \boldsymbol{\bullet} \\
& -\frac{1}{6}(\alpha h)^{3} \cdot x^{\prime \prime \prime}[n]
\end{aligned}
$$

Clearly, the derivatives of (14) shall be approximated and expressed as function of the asynchronous samples set $x[n]$ " In such a way, each synchronous sample can be computed using asynchronous samples. The approximation can be performed through the use of the finite difference methods [33].

Applying a backward finite difference on (14) yields

$$
\begin{gathered}
x[n]=b_{o} x[n] \mathbf{\bullet}+b_{1} x[n-1]^{\bullet}+b_{2} x[n-2] \boldsymbol{\bullet} \\
+b_{3} x[n-3]
\end{gathered}
$$

where

$$
\begin{gathered}
b_{o}=1-\frac{11}{6} \alpha+\alpha^{2}-\frac{1}{6} \alpha^{3} \\
b_{1}=3 \alpha-2.5 \alpha^{2}+0.5 \alpha^{3} \\
b_{2}=-1.5 \alpha+2 \alpha^{2}-0.5 \alpha^{3} \\
b_{3}=\frac{1}{3} \alpha-0.5 \alpha^{2}+\frac{1}{6} \alpha^{3}
\end{gathered}
$$

Equation (15) shows that to resample $N$ samples, $(N+3)$ samples need to be stored. It should be noted also that higher order accuracies will require a bigger buffer.

Moreover, when $n=0$, all the coefficients disappear except $b_{0}$ which is equal to 1 , this means $x[0]=x[0]^{\mathbf{*}}$. In other words, the synchronous sample is exactly the same as the asynchronous sample when $n=0$ while other resampled data are affected by variable coefficients, altering their amplitudes. Thus, the phase angle information of the asynchronous sample set $x[n]^{\bullet}$, computed through the DFT, is kept after resampling only for $n=0$.

Reference [30] has applied backward finite difference coefficients on the whole observation window, taking the most recent sample of that window as it is. Consequently, the resampling strategy, named Sampled Value Adjustment (SVA), has been performed from the most recent sample to the oldest one (time-reversal), for $n=0,-1,-2, \ldots-N$, where $x[0]$ denotes the newest sample. Based on that, to extract the correct phase angle, the Full-Cycle DFT should be applied as follows

$$
X_{0}=\frac{\sqrt{2}}{N} \sum_{n=-N+1}^{0} x[n] e^{-j \frac{2 \pi}{N} n}
$$

The previous SVA method is suitable for protective relay purpose where there is no need to neither synchronize the DFT estimates with an absolute time reference nor center the observation window at the synchrophasor reporting time. However, unlike its predecessor, the IEEE Standard C37.118.1-2011 advises to timestamp at the middle of a given observation window, i.e. the phase angle of the synchrophasor to be reported is centered on the window length. Therefore, the response time of the PMU will fall within the limits imposed. In conclusion, it is not possible to follow the method described in [30] where the timestamp is placed at the end of the observation window.

The idea of our design is to displace the window in such a way that the timestamp is near the center of that window while keeping the correct phase angle given by $x[0]^{\text {" }}$. Actually, since $N$ is even, it is not possible for $x[0]$ ' to be exactly at the center of a given data window as shown in Fig. 2. A delay of half a sampling period is introduced. However, this delay is not critical neither for the synchrophasor response time nor the delay time. Therefore, to cope with the new synchrophasor dynamic performances, the magnitude of the sample $x[0]$ " shall remain unchanged after the resampling process. To do so, in addition to the backward computation, the proposed algorithm uses the forward finite difference methods whose general equation could be written as

$$
\begin{aligned}
x[n]=b_{n} x[n] & +b_{n+1} x[n+1]^{\mathbf{\bullet}}+b_{n+2} x[n+2]^{\mathbf{}} \\
& +b_{n+3} x[n+3]^{\bullet}
\end{aligned}
$$

Thus, for a given synchrophasor reporting time, the data of the window on the left of the synchrophasor time-tag (see time-tag $t_{\text {tag }}=0.04 \mathrm{~s}$ in Fig. 2) will be resampled following the backward method described above while the samples on the right will be re-computed using forward finite difference. This new principle is called enhanced-SVA (e-SVA).

Basically, the coefficients of the forward finite difference are obtained by giving the opposite sign (-) to all backward coefficients of odd derivatives, which are $x^{\prime}[n]$ ' and $x^{\prime \prime \prime}[n]$ " in our case. However, such a rule will unequivocally lead to changes in the coefficients defined in (16) - (19) making them unusable for the forward finite difference as shown below

$$
\begin{aligned}
& b_{o}=1+\frac{11}{6} \alpha+\alpha^{2}+\frac{1}{6} \alpha^{3} \\
& b_{1}=-3 \alpha-2.5 \alpha^{2}-0.5 \alpha^{3} \\
& b_{2}=1.5 \alpha+2 \alpha^{2}+0.5 \alpha^{3} \\
& b_{3}=-\frac{1}{3} \alpha-0.5 \alpha^{2}-\frac{1}{6} \alpha^{3}
\end{aligned}
$$

Through the e-SVA method, the resampling is mirrored on both sides of the timestamp, which means that the reverse 
computation with backward finite difference coefficients could be seen as a computation following the time axis with forward coefficients. Indeed, substituting $\alpha$ by $-\beta$ in (16) (19) leads to the same coefficients given by (22) $-(25)$. The only difference for the backward computation lies on the values of $n$ in the expression $\alpha=n \Delta f$ which has to be given with the opposite sign, that is $\beta=-n \Delta f$. As a result, (22)(25) are valid in both cases and are used in our algorithm.

Following the above discussion, considering that $N$ is an even integer, the time-reversal backward resampling is applied for $n=0,-1,-2, \ldots-\frac{N}{2}$ while the forward resampling is done for $n=0,1,2, \ldots \frac{N}{2}-1$. The size of the buffer is equal to $(N+6)$, where 3 more samples are necessary for each resampling method as shown in (15) and (21). An example of e-SVA method applied on the signal $x(t)=\cos (2 \pi(45) t)$, sampled at a sampling rate of $f_{s 0}=24 \times 50 \mathrm{~Hz}$, at a synchrophasor time-tag $t_{\text {tag }}=0.04 \mathrm{~s}$, is shown in Fig. 2 .

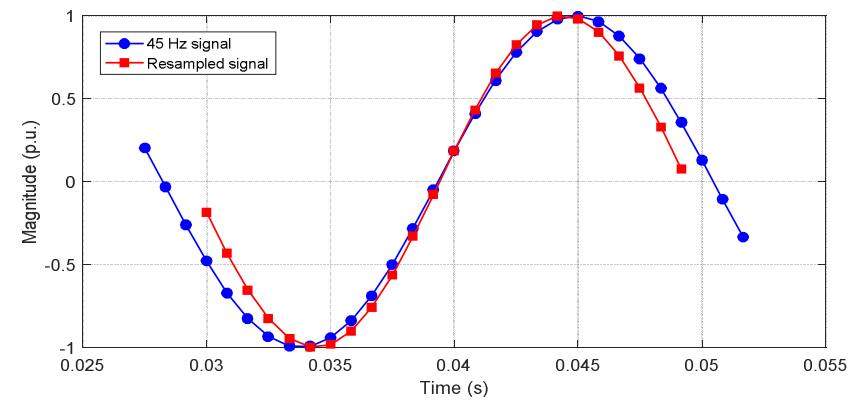

Fig. 2. e-SVA method of a $45 \mathrm{~Hz}$ signal, at $\mathrm{t}_{\mathrm{tag}}=0.04 \mathrm{~s}$.

Clearly, the amplitude of the sample $n=0$ at $t_{t a g}=0.04 \mathrm{~s}$ remains unchanged while the other asynchronous samples are re-computed in such a way that the resampled signal appears as a synchronous $50 \mathrm{~Hz}$ signal.

The previous discussion is applicable only for frequencies below the nominal value. The computation is slightly different for off-nominal frequencies above the nominal. Following the same reasoning described in [30], for frequencies higher than $50 \mathrm{~Hz}$, considering $\alpha$ as $\left[n\left(\frac{f-f_{o}}{f}\right)+2\right]$ gives better accuracy.

Finally, another important point to mention concerns the computation of the Full-Cycle DFT whose coefficients have to be adapted to extract the correct phase angle information from the resampled signal. Consequently, (18) has been modified as shown in (26).

$$
X_{0}=\frac{\sqrt{2}}{N} \sum_{n=-\frac{N}{2}}^{\frac{N}{2}-1} x[n] e^{-j \frac{2 \pi}{N} n}
$$

\section{Frequency Estimation Algorithm}

As described in the previous sections, resampling technique requires knowledge of power system frequency. A lot of methods with regards to frequency estimation such as the techniques that have been published in the literature [34]-[37] exist. But most of them require high computational burden and complex implementation. This paper has integrated the
Fourier algorithm frequency estimation with the proposed resampling algorithm as it is relatively simple, computationally efficient and can also estimate the fundamental frequency accurately [38]. Fourier algorithm for frequency estimation is based on the phase angle difference between two phasors. The samples of the voltage waveforms are preferred to be used to estimate the frequency since the harmonics distortion in the voltage is less than harmonics distortion in the current. Also it can be shown that the effect of the $Q$ factor defined in (8) can be removed using the positive sequence of balanced three phase voltages [39]. Based on the above assumptions, (6) can be rewritten as

$$
X^{\prime}{ }_{r}=P \cdot X_{0} e^{j r \omega \Delta t_{o}}
$$

The phase angle of $X^{\prime}{ }_{r}$ in (27) is

$$
\phi_{r}^{\prime}=r \omega \Delta t_{o}+(N-1)\left(\frac{\omega-\omega_{0}}{2}\right) \Delta t_{o}
$$

The phase angle difference between $X^{\prime}{ }_{r+n}$ and $X^{\prime}{ }_{r}$ can be given by (29).

$$
\phi_{r+n}^{\prime}-\phi_{r}^{\prime}=\Delta \phi_{r}^{\prime}=\frac{2 \pi n}{f_{o} N} f
$$

Based on (29), the frequency can be estimated using (30).

$$
f=f_{o} \cdot\left(\frac{\Delta \phi_{r}^{\prime}}{\left(\frac{2 \pi n}{N}\right)}\right)
$$

Equation (30) estimates the frequency with high accuracy only for a positive sequence of three phase balanced system with no harmonics. Usually this is not the case, because harmonics do exist in power systems and these latter may not always operate under fully balanced conditions due to unbalance loads. That is why there will be an error in the frequency estimation based on Fourier algorithm. To solve this problem, the frequency estimation algorithm should be used in a closed loop with the resampling algorithm. In this case the error will be minimized till the frequency estimation converges to the correct value.

The accuracy of resampling depends on the precision with which the frequency is estimated. Inaccurate resampling result in wrong phasor measurements. However, as shown in Section $\mathrm{V}$, errors of the order of few millihertz have small impact on the estimation of synchrophasors.

\section{Computational Effort}

An analysis of the computational effort is necessary to understand whether the method can be integrated into a protection relay. To do so, each basic operation (addition and multiplication) to perform has been counted for the e-SVA, the Full-Cycle DFT, and the frequency estimation algorithms under both the ideal and the worst conditions. Actually, the computational effort is dependent on the frequency estimates as they drive the e-SVA resampling algorithm which is executed if the frequency is off-nominal. Thus, the ideal scenario refers to a nominal power system frequency under 
which the use of the e-SVA algorithm is omitted. On the other hand, the computation burden is maximal if the frequency estimation algorithm measures a frequency above the nominal as $\alpha$ contains one more addition in contrast to its expression when the frequency is under the nominal. The Full-Cycle DFT in (26) is performed only for the fundamental frequency making it CPU-efficient. As the positive sequence component is required to measure the frequency, (26) is applied in the same manner to the three-phase voltages and the positive sequence is then computed. Finally, as $f_{o}, N$, and $n$ are fixed once the sampling frequency and the number of samples between two consecutive phasors $n$ are chosen, (30) can be reduced to a simple multiplication and one subtraction making the algorithm ideal for practical implementation. The value of $\Delta \phi_{r}^{\prime}$ can be computed through the arctangent operation. Table I provides the detailed number of operations to perform for $N$ samples under ideal and worst conditions. Obviously, the way the algorithm is implemented may change the number of operations reported in Table I.

\section{TABLE I}

Computational effort of the proposed method for $\mathrm{N}$ samples

\begin{tabular}{|l|l|c|c|}
\cline { 3 - 4 } \multicolumn{2}{c|}{} & $\begin{array}{c}\text { Ideal } \\
\text { Condition }\end{array}$ & $\begin{array}{c}\text { Worst } \\
\text { Condition }\end{array}$ \\
\hline \multirow{2}{*}{ e-SVA } & Addition/Subtraction & 0 & $14 \times \mathrm{N}$ \\
\cline { 2 - 4 } & Multiplication & 0 & $27 \times \mathrm{N}$ \\
\hline \multirow{2}{*}{$\begin{array}{l}\text { Full-Cycle DFT } \\
\text { for each phase) }\end{array}$} & Complex Addition & $\mathrm{N}-1$ & $\mathrm{~N}-1$ \\
\cline { 2 - 4 } $\begin{array}{l}\text { Positive Sequence } \\
\text { Calculation }\end{array}$ & Complex Multiplication & $\mathrm{N}$ & $\mathrm{N}$ \\
\cline { 2 - 4 } $\begin{array}{l}\text { Frequency } \\
\text { Estimation }\end{array}$ & Complex Addition & 2 & 2 \\
\cline { 2 - 4 } & Complex Multiplication & 2 & 2 \\
\cline { 2 - 4 } & Multiplication & 1 & 1 \\
\hline
\end{tabular}

As shown in Table I, the complexity of the whole algorithm is $O(n)$ : the number of elementary operations increases linearly with the number of sample $N$. For $N=24$, the computational effort for the ideal condition consists of 25 complex additions, 26 complex multiplications, 1 addition and 1 multiplication. If the frequency is above the nominal, then the e-SVA algorithm introduces 336 additions/subtractions and 648 multiplications.

\section{E. General Overview of the Proposed Solution}

The analog signal is directed to a low pass filter and then to an ADC. Depending on its design, the analog low pass filter may change the amplitude of the analog input signal and/or introduces a phase shift. Consequently, compensation methods are necessary for correct synchrophasor estimations. The analog low pass filter is a $2^{\text {nd }}$ order Butterworth filter with a cutoff frequency of $500 \mathrm{~Hz}$. The frequency response of such a filter is depicted in Fig. 3 for the frequency range from DC to $100 \mathrm{~Hz}$ to ease the analysis following.
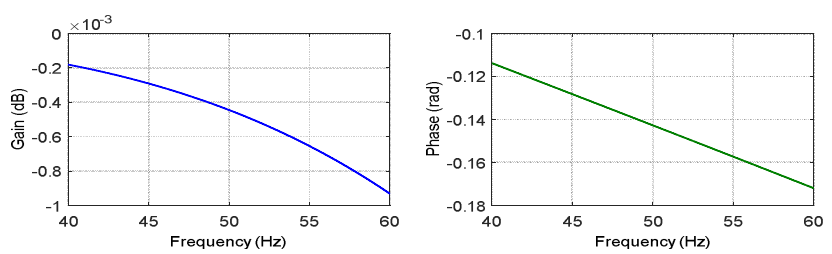

Fig. 3. Frequency response of the $2^{\text {nd }}$ order Butterworth filter.
As shown in Fig. 3, the magnitude response of the $2^{\text {nd }}$ order Butterworth filter is flat and very near $0 \mathrm{~dB}$ while its phase response is almost perfectly linear within the frequency range of interest. Thus, the impact of the magnitude response on the synchrophasor estimates may be neglected. Moreover, the phase shift introduced can be corrected simply by estimating the actual power system frequency through the frequency estimation algorithm described in Section IV.C and multiplying the frequency estimate by the slope preceded by the negative sign.

The ADC samples the signal at a specific selected sampling interval synchronized to the nominal frequency thanks to the absolute time reference GPS. The samples obtained are then forwarded to the e-SVA algorithm which alters the amplitude of the sample sequence based on the estimated frequency. The modified samples are then used for synchrophasor estimation through the DFT as shown in Fig. 4.

Initially, it is assumed that the power system frequency is nominal. If a $N$-point DFT is performed once every $n$ samples, then $N+n$ samples are needed at the beginning to estimate two consecutive phasors in order to estimate the power system frequency. If the estimated frequency is off-nominal, then the sampled signal will be resampled at a rate which is synchronized with the off-nominal power system frequency. In our proposal, we assume that $n$ is equal to $N / 2$, which means that the two consecutive phasors are delayed by $N / 2$ samples.

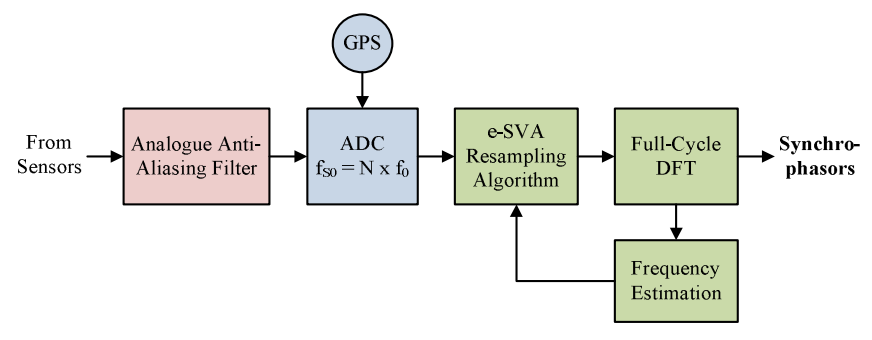

Fig. 4. General overview of the proposed solution.

\section{Simulation Results}

This section presents the simulation and result analysis of P-Class synchrophasor measurements for a power system with $50 \mathrm{~Hz}$ nominal frequency. The TVE and the Frequency Error (|FE|) defined in [31] have been chosen as criteria of assessment to demonstrate the performances of the e-SVA method and the frequency estimation algorithm under both steadystate and dynamic conditions. To do so, the proposed technique has been implemented using the MATLAB environment.

The IpDFT algorithm of [21] has been chosen as indicator for comparison as the technique gives accurate results with TVE below the $1 \%$ criterion for the P-Class test cases. Table II provides a quick comparison of the proposed method against the IpDFT algorithm of [21] where the 2 cycles window length version has been selected to cope with P-Class PMU requirements. 
TABLE II

Quick comparison between the proposed method and IpDFT

\begin{tabular}{|l|c|c|}
\hline & $\begin{array}{c}\text { Proposed } \\
\text { Method }\end{array}$ & IpDFT \\
\hline Interpolation domain & Time & Frequency \\
\hline Sampling rate (kHz) & 1.2 & 10 \\
\hline Window length (samples) & 30 & 400 \\
\hline Computational burden (basic operations) & 1035 & 3614 \\
\hline Tapering function & N/A & Hanning \\
\hline
\end{tabular}

Compared to the IpDFT algorithm, our proposed method interpolates the signal in the time domain rather than in the frequency domain. Also, neither windowing functions nor high sampling rates are required. Furthermore, the window length considered for the e-SVA algorithm is equal to just 30 samples (the e-SVA algorithm requires 30 samples to compute 24 resampled samples), making the synchrophasor estimation algorithm response time better than that of algorithms based on longer windows. Finally, as the number of samples is low and the use of tapering functions is unnecessary through the eSVA method, the number of basic operations to perform is more than 3 times lower than that of the IpDFT technique.

Unlike [21], the accuracy with which the IpDFT algorithm estimates the power system frequency is also presented through the $|\mathrm{FE}|$ criterion and compared with our proposed method. The test cases conducted cover the IEEE Standard C37.118.1-2014a test procedure for:

(A) Steady-State with off-nominal frequencies,

(B) Steady-State with harmonic distortions

(C) Amplitude and phase modulations,

(D) Positive and negative ramps of frequency,

The response time of the frequency estimation algorithm is provided in $(\mathrm{E})$.

The amplitude of the input signals is 1 p.u. Without loss of generality, the TVE have been reported for the phase A. Finally, a sliding window is considered in the simulation results which means that a new synchrophasor estimate and a new frequency estimate is computed at each new sample.

\section{A. Steady-State with Off-Nominal Frequencies}

Simulation details:

- Steady-State off-nominal frequency range: [48; 52] Hz, with a steepness of $0.1 \mathrm{~Hz}$,

- Duration of simulation: $5 \mathrm{~s}$,

- Maximum TVE: $1 \%$,

- Maximum |FE|: $0.005 \mathrm{~Hz}$.

The tests have been conducted on the whole frequency range described above where the maximum TVE, the average TVE, and the minimum TVE values have been computed for each case. The results have been summarized in Fig. 5 where the "Average" curve is almost superposed with the "Min" curve. As shown in Fig. 5, the TVE is far away from the fixed limit of $1 \%$ on the whole range of off-nominal frequencies for both methods even if e-SVA shows more accurate results. Furthermore, the $|\mathrm{FE}|$ values computed for IpDFT are above the standard threshold while our proposed method is able to estimate the frequency with error near $0 \%$.

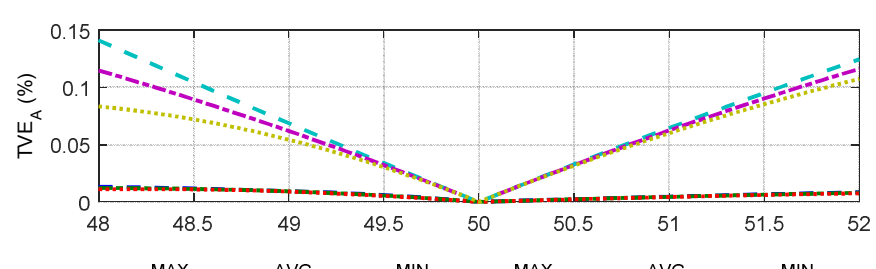

- $-\operatorname{MAX}_{\mathrm{SVA}}=-\mathrm{AVG}_{\mathrm{SVA}} \ldots . . . \mathrm{MIN}_{\mathrm{SVA}}=-\mathrm{MAX}_{\mathrm{IPDFT}}--\mathrm{AVG}_{\mathrm{IPDFT}} \ldots \ldots \mathrm{MIN}_{\mathrm{IPDFT}}$

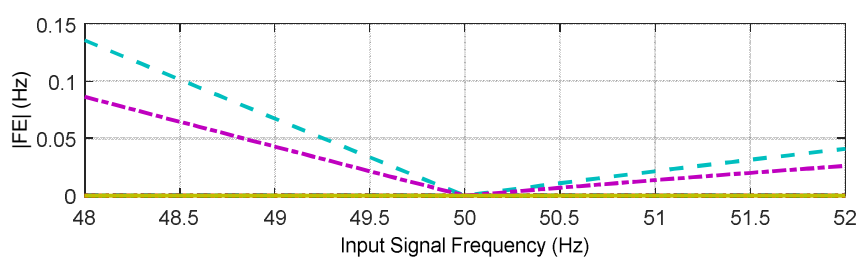

Fig. 5. TVE under steady-state off-nominal frequencies.

\section{B. Steady-State with harmonic distortions}

Simulation details:

- Harmonic level: $1 \%$

- Harmonic range: $\left[2^{\text {nd }} ; 50^{\text {th }}\right]$, with a steepness of 1 ,

- Duration of simulation: $5 \mathrm{~s}$,

- Maximum TVE: $1 \%$,

- Maximum |FE|: $0.005 \mathrm{~Hz}$.

The input signals are corrupted with a single harmonic ranging from the $2^{\text {nd }}$ to the $50^{\text {th }}$ harmonic. The results are shown in Fig. 6.

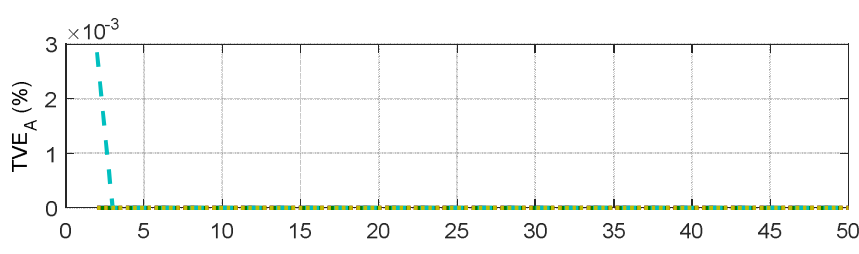

$$
\text { - }-\mathrm{MAX}_{\mathrm{SVA}}=-\mathrm{AVG}_{\mathrm{SVA}} \ldots \ldots \mathrm{MIN}_{\mathrm{SVA}}--\mathrm{MAX}_{\mathrm{IPDFT}}---\mathrm{AVG}_{\mathrm{IPDFT}} \ldots \ldots \mathrm{MIN}_{\mathrm{IPDFT}}
$$

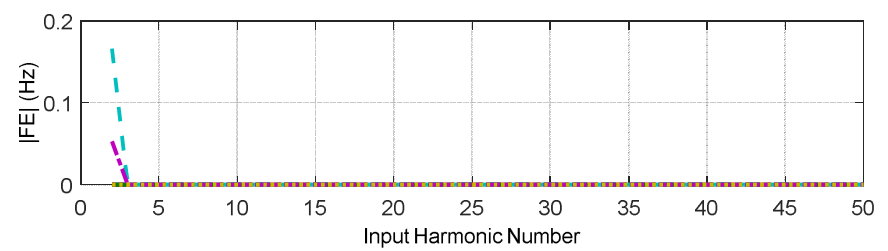

Fig. 6. TVE under steady-state with harmonic distortions.

As shown is Fig. 6, both methods give satisfactory results for the whole harmonic range with error near $0 \%$. However, when the signal is corrupted by the $2^{\text {nd }}$ harmonic, the IpDFT becomes less accurate than results obtained with other harmonic contents. Indeed, in this condition, the errors introduced in the frequency estimates are above the standard limit of $0.005 \mathrm{~Hz}$.

\section{Amplitude and Phase Modulations}

Simulation details:

- Initial frequency: $50 \mathrm{~Hz}$,

- Modulation frequency range: $[0.1 ; 2] \mathrm{Hz}$, in steps of $0.1 \mathrm{~Hz}$,

- Amplitude modulation level: 0.1 p.u.,

- Phase modulation level: $0.1 \mathrm{rad}$.,

- Duration of the simulation: 2 full cycles of modulation, 
- Maximum TVE: $3 \%$,

- Maximum |FE|: $0.06 \mathrm{~Hz}$.

According to [31], the amplitude and phase modulation tests are realized separately, which means that the modulation signal shall contain either the amplitude factor or the phase factor. The results on the phase A are depicted in Fig. 6 (a) and Fig. 6 (b).
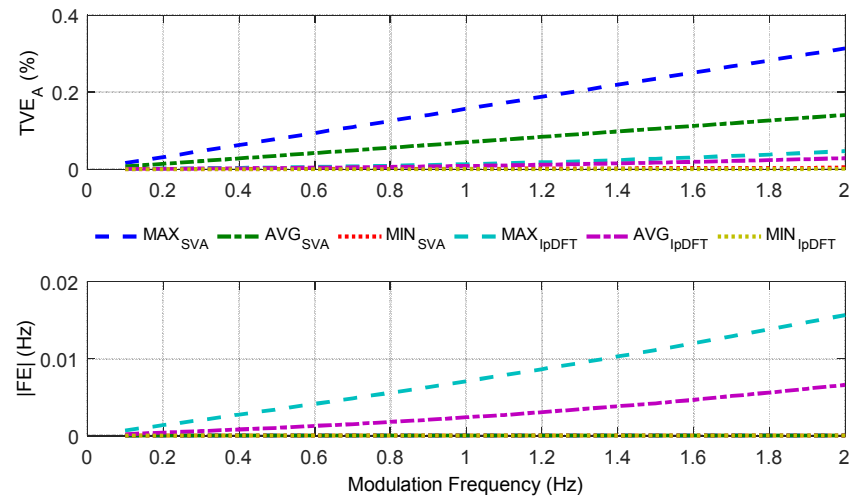

(a)

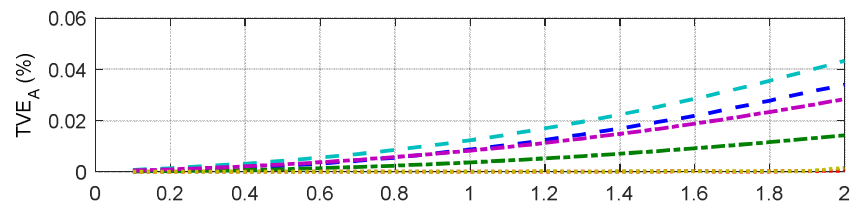

$--\mathrm{MAX}_{\mathrm{SVA}}=-\mathrm{AVG}_{\mathrm{SVA}} \ldots \ldots \mathrm{MIN}_{\mathrm{SVA}}=-\mathrm{MAX}_{\mathrm{IPDFT}}---\mathrm{AVG}_{\mathrm{IPDFT}} \ldots \ldots \mathrm{MIN}_{\mathrm{IPDFT}}$

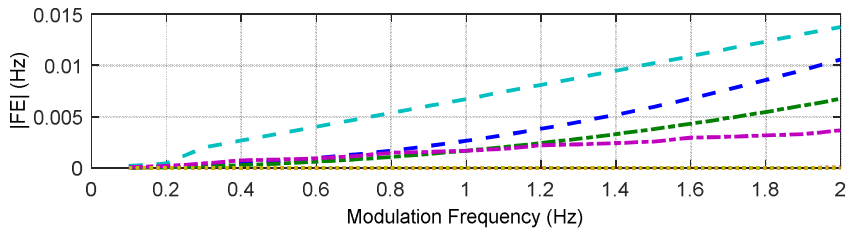

(b)

Fig. 6. (a) TVE under amplitude modulation. (b) TVE under phase modulation.

As we can see from Fig. 6 (a) and Fig. 6 (b), the accuracies of synchrophasor estimates and frequency estimates are satisfactory as the TVE and the $|\mathrm{FE}|$ are below the standard thresholds. The worst cases occur when the $50 \mathrm{~Hz}$ signal is modulated with a modulation frequency of $2 \mathrm{~Hz}$. Regarding the TVE, the e-SVA method gives better results than IpDFT for phase modulations whereas the opposite is true for amplitude modulations. Moreover, the frequency estimations realized through our proposal are more accurate than IpDFT under amplitude modulation while IpDFT is less sensitive in case of phase modulation to estimate the frequency. Finally, despite the fact that some errors are introduced in the frequency estimated by our proposal, the TVE remains below $1 \%$ showing that the impact of errors in frequency measurements on the TVE is minor.

\section{Positive and Negative Ramps of Frequency}

Simulation details:

- Initial frequencies: $48 \mathrm{~Hz}$, and $52 \mathrm{~Hz}$ for positive and negative ramps respectively,
- Ramp rates: +/- $1.0 \mathrm{~Hz} / \mathrm{s}$, for positive and negative ramps respectively,

- Ramp ranges of frequency: [48; 52] Hz, and [52; 48] $\mathrm{Hz}$, for positive and negative ramps respectively,

- The ramps are applied at $\mathrm{t}=1.0 \mathrm{~s}$,

- Simulation stops at $\mathrm{t}=5 \mathrm{~s}$,

- Maximum TVE: $1 \%$,

- Maximum |FE|: $0.01 \mathrm{~Hz}$.

The results for both positive and negative ramps of frequency are drawn in Fig. 7 (a) and Fig. 7 (b) respectively, where the TVE and the $|\mathrm{FE}|$ have been computed once each new sample during the whole simulation duration starting at $t=0.04 \mathrm{~s}$.
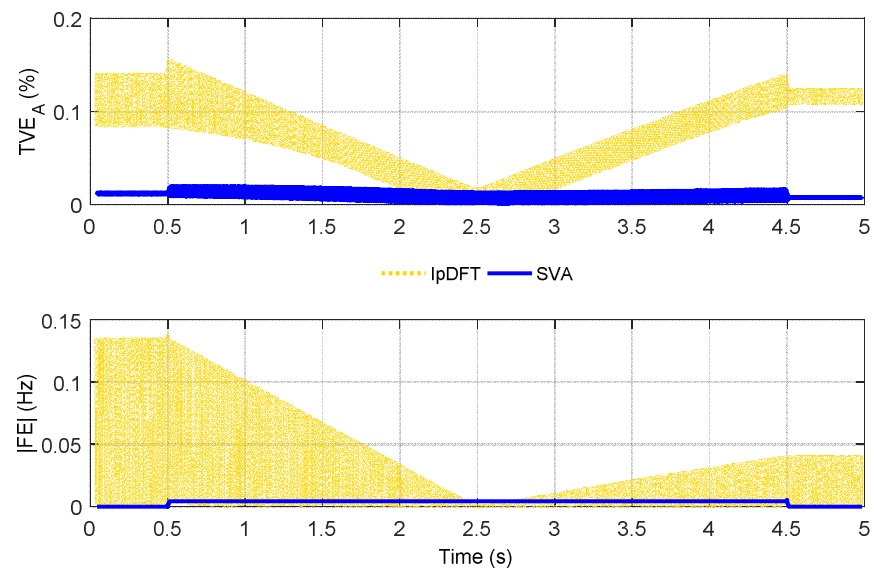

(a)
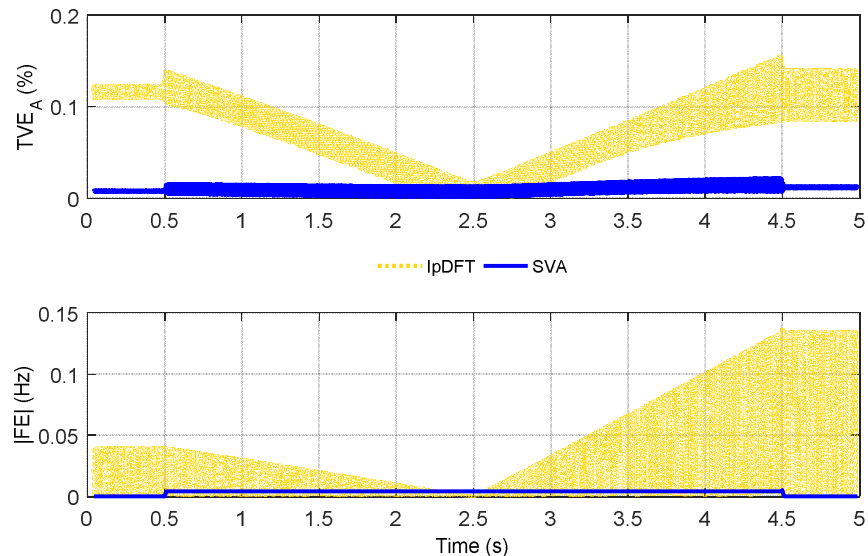

(b)

Fig. 7. (a) TVE under positive ramp of frequency. (b) TVE under negative ramp of frequency.

As depicted in Fig. 7 (a) and Fig. 7 (b), the TVE values remain below the $1 \%$ criterion for both cases, demonstrating that the e-SVA method and the IpDFT algorithm are robust in case of ramps of frequency for P-Class PMU. However, the TVE values fluctuate within a wide range regarding IpDFT and are dependent on the instant when the computation is realized. This remark is especially true for frequency estimates where fluctuations vary between almost $0 \mathrm{~Hz}$ and $0.136 \mathrm{~Hz}$ when the power system frequency is equal $48 \mathrm{~Hz}$. Conversely, the e-SVA seems to be less sensitive to the instant of computation and the maximum $|\mathrm{FE}|$, measured during the 
tracking, is $0.0042 \mathrm{~Hz}$. Here also, small errors in frequency estimates do not affect significantly the TVE.

\section{E. Frequency Estimation Response Time}

The input signal is held at $50 \mathrm{~Hz}$ up till $1 \mathrm{~s}$. Then, the frequency jumps from $50 \mathrm{~Hz}$ to $55 \mathrm{~Hz}$ at $\mathrm{t}=1 \mathrm{~s}$. Finally, the signal remains at $55 \mathrm{~Hz}$ up till $2 \mathrm{~s}$. The time response of the frequency estimation algorithm is shown in Fig.8.

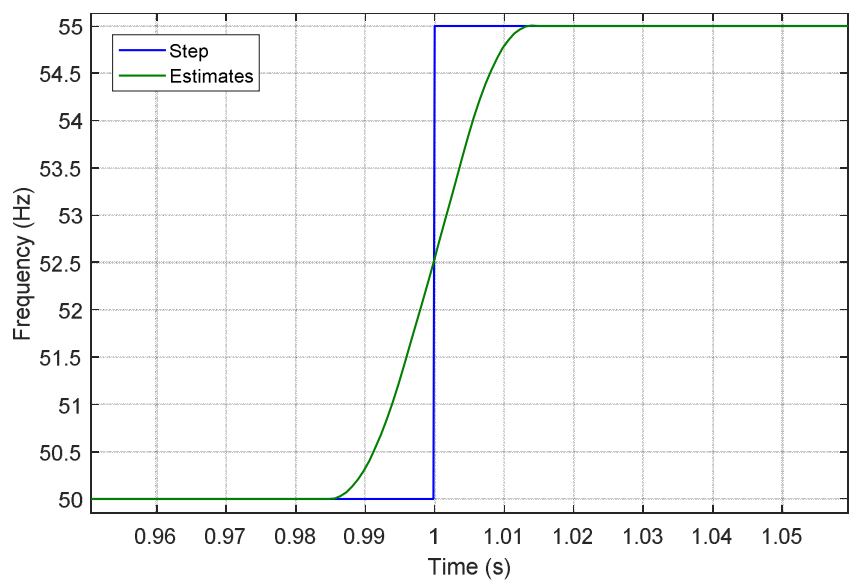

Fig. 8. Response time of the frequency estimation algorithm.

As shown in Fig. 8, the duration necessary to converge to the expected value is equivalent to the window length of 30 samples obtained by sampling the input signal at a rate of 1.2 $\mathrm{kHz}$ and required to measure the frequency. One important point to highlight is that no convergence criterion has been implemented: once a new frequency is estimated, it is then used directly by the e-SVA algorithm during the next execution cycle (closed-loop). Thus, only 1 iteration is necessary to obtain 1 frequency estimate value.

\section{CONCLUSION}

In this work, the leakage problem of the DFT caused by the asynchronous sampling has been discussed. The model that explains how the error is introduced to the DFT estimate has been presented and has been used to derive the frequency estimation algorithm. A solution to the DFT leakage problem has been proposed. The proposed solution alters the sample value amplitudes in order to generate new set of samples whose sampling frequency matches the power system frequency, which results in accurate synchrophasor estimation and frequency estimates under various conditions, challenging the widespread IpDFT methods in terms of accuracy, sampling rate, transient response, and computational effort. The simulation results, conducted following the IEEE Standard C37.118.1-2014a for P-Class PMU, show that the proposed solution is compliant with P-Class PMU accuracy class.

\section{REFERENCES}

[1] A. G. Phadke "Precise synchronization of phasor measurements in electric power systems", Twenty-Second Annual Precise Time and Time Interval (PTTI) Applications and Planning Meeting, 1990.

[2] A. G. Phadke "The wide world of wide-area measurement", IEEE Power Energy Mag., vol. 6, no. 5, pp.52 -65 2008.
[3] Qi Huang, Shi Jing, Jianbo Yi and Wei Zhen "Innovative Testing and Measurement Solutions for Smart Grid". Singapore: John Wiley \& Sons, 2015. Chapter 3.

[4] A. G. Phadke and J. S. Thorp "Computer Relaying for Power Systems", U.K.: Research Studies Press, 1990.

[5] G. Benmouyal, E. O. Schweitzer, and A. Guzman "Synchronized phasor measurement in protective relays for protection, control, and analysis of electric power systems," in Proc. 57th Annu. Conf. Protective Relay Engineers, 2004, pp. 419-450.

[6] A. G. Phadke and J. S. Thorp "Synchronized Phasor Measurements and Their Applications". New York: Springer Science, 2008, pp. 4978. Chapter 3 (section 3.7).

[7] D. Fan and V. Centeno "Least-squares estimation in phasor-based synchronized frequency measurements" in Proc. IEEE Power and Energy Society General Meeting-Conversion and Delivery of Electrical Energy in the 21st Century, 2008, pp. 1-6.

[8] I.Kamwa, S.R.Samantaray, and G.Joos "Wide frequency range adaptive phasor and frequency PMU algorithms", IEEE Trans. Smart Grid, vol. 5, no. 2, pp. 569-579, Mar. 2014.

[9] A. G. Phadke "Synchronized Phasor Measurements - A Historical Overview", in Proc. IEEE/PES Transmission and Distribution Conf. 2002, vol. I, pp.476-479.

[10] Jan Machowski, James Richard Bumby "Power System Dynamics and Stability" New York: John Wiley \& Sons, 1997. Chapter 8

[11] Saeed V. Vaseghi. "Multimedia Signal Processing Theory and Applications in Speech", Music and Communications. New York: John Wiley \& Sons, 2007. Chapter 2.

[12] G. Benmouyal "An adaptive sampling interval generator for digital relaying”, IEEE Trans. Power Del., vol. 4, no. 3, pp.1602 -1609 1989.

[13] G. Benmouyal "System and algorithm for exact compensation of fundamental phasors" U.S. Patent 6,934,654, Aug. 23, 2005.

[14] J. Z. Yang and C. W. Liu "A Precise Calculation of Power System Frequency and Phasor", IEEE Trans. Power Del., vol. 15, no. 2, pp.494 -499 2000

[15] Joseph P. Benco, Germansville; Frederick P. Perfect, Monthon, both of Pa.; David G. Hart;JamesD. Stoupis, both of Raleigh, N.C, "Generator Protection System and Method for Phasor Estimation and Frequency Tracking During frequency Ramping" U.S. Patent 5,832,413, issued Nov. 3, 1998.

[16] T. T. Nguyen and X. J. Li "A fast and accurate algorithm for estimating power systems phasors using DFT with interpolation" in Proc. Power Eng. Soc. General Meet., 2006, pp. 1-8

[17] F. J. Harris "On the use of windows for harmonic analysis with the Discrete Fourier Transform” Proc. of the IEEE, vol. 66, pp. 51-83. Jan. 1978

[18] V. K. Jain, W. L. Collins and D. C. Davis, "High-accuracy analog measurements via interpolated FFT", IEEE Trans. Instrum. Meas., vol. IM-28, pp. 113-122, 1979

[19] T. Grandke, "Interpolation algorithms for discrete Fourier transforms of weighted signals", IEEE Trans. Instrum. Meas., vol. 32, no. 2 , pp. 350-355, Jun. 1983.

[20] IEEE Standard for Synchrophasor Measurements for Power Systems, IEEE Standard C37.118.1-2011, Dec. 2011.

[21] D. Belega and D. Petri, "Accuracy of the synchrophasor estimator provided by the interpolated DFT algorithm", Proc. IEEE Int. Instrum. Meas. Technol. Conf., pp. 1-6, 2012

[22] D. Belega and D. Petri, "Accuracy analysis of the multi-cycle synchrophasor estimator provided by the interpolated DFT algorithm", IEEE Trans. Instrum. Meas., vol. 62, pp. 942-953, 2013

[23] D. Belega, D. Macii and D. Petri, "Fast synchrophasor estimation by means of frequency-domain and time-domain algorithms", IEEE Trans. Instrum. Meas., vol. 63, no. 2, pp. 388-401, 2014

[24] P. Romano and M. Paolone, "Enhanced interpolated-DFT for synchrophasor estimation in FPGAs: Theory, implementation, and validation of a PMU prototype", IEEE Trans. Instrum. Meas.,vol. 63, no. 12,2014

[25] P. Romano, M. Paolone, J. Arnold and R. Piacentini, "An interpolated-DFT synchrophasor estimation algorithm and its implementation in an FPGA-based PMU prototype", Proc. IEEE PES General Meeting, pp. 1-6

[26] P. Romano, M. Paolone, "An enhanced interpolated-modulated sliding DFT for high reporting rate PMUs," presented at the International workshop on Applied Measurements for Power Systems (AMPS), Aachen, Germany, 24-26 Sept. 2014 
[27] A. G. Phadke and J. S. Thorp "Synchronized phasor measurements and their applications", New York: Springer, 2008. Chapter 3 (section 3.3.2)

[28] J. Z. Yang and C. W. Liu "A Precise Calculation of Power System Frequency”, IEEE Trans. Power Del., vol. 16, no. 3, pp.361 -366 2001

[29] J. Xi and J. F. Chicharo "A new algorithm for improving the accuracy of periodic signal analysis," IEEE Trans. Instrum. Meas., vol. 45, no. 4, pp. 829-831, Aug. 1996.

[30] M. Akke, J.S. Thorp "Sample Value Adjustment Improves Phasor Estimation at Off-Nominal Frequencies" IEEE Trans. Power Del., Vol. 25, No. 4, Oct. 2010.

[31] IEEE Standard for Synchrophasor Measurements for Power Systems, IEEE Standard C37.118-2005, April 2006.

[32] IEEE Standard for Synchrophasor Measurements for Power Systems, IEEE Standard C37.118.1a-2014, March 2014.

[33] Jaan Kiusalaas "Numerical Methods in Engineering with MATLAB" New York: Cambridge University Press, 2010. Chapter 5.

[34] Terzija, V.V., Djuric, M.B., and Kovacevic, B.D. "Voltage phasor and local system frequency estimation using Newton-type algorithms" IEEE Transactions on Power Delivery, Vol. 9, No. 3, 1994, pp 13681374.

[35] Sidhu, T.S. and Sachdev, M.S. "An iterative technique for fast and accurate measurement of power system frequency", IEEE Trans. Power Del., Vol. 13, No.1, 1998, pp 109-115.

[36] Girgis, A.A. and Hwang, T.L.D. "Optimal estimation of voltage phasors and frequency deviation using linear and non-linear Kalman filtering", IEEE Transactions on Power Apparatus and Systems, Vol. 103, No. 10, 1984, pp 2943-2949.

[37] Moore, P.J., Carranza, R.D., and Johns, A.T. "A new numeric technique for high-speed evaluation of power system frequency", IEEE Proceedings Generation, Transmission and Distribution, Vol. 141, No. 5, 1994, pp 529-536.

[38] M. Wang and Y. Sun "A practical, precise method for frequency tracking and phasor estimation", IEEE Trans. Power Del., vol. 19, no. 4, pp. $1547-15522004$

[39] A. G. Phadke and J. S. Thorp "Synchronized Phasor Measurements and Their Applications". New York: Springer Science, 2008, pp. 4978. Chapter 3 (section 3.4.1).

\section{BIOGRAPHIES}

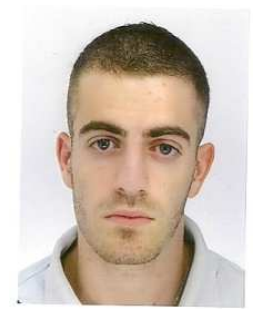

Christophe Ghafari was born in Grenoble in France, on December 18, 1990. He received his M.Sc. degree in electrical engineering from the Institut National Polytechnique de Grenoble (INPG) at the Ecole Nationale Supérieure de l'Energie, de l'Eau et de l'Environnement (ENSE3) in Grenoble, France, in 2013. Currently, he is pursuing a Ph.D. degree at the Grenoble Electrical Engineering laboratory (G2Elab), Grenoble, France. His general research interests are centered on power system protective relaying, digital signal processing for Intelligent Electronic Devices, and protection algorithms.

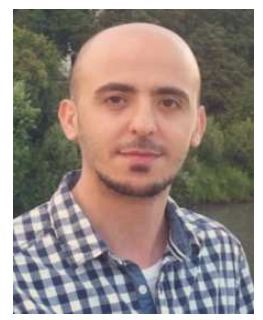

Hamada Almasalma received the B.Sc. degree in electrical engineering from Birzeit University, Birzeit, Palestine, in 2011 and the M.Sc. degree in smart grids from Grenoble Institute of Technology (Ecole Nationale Supérieure de l'Energie, de l'Eau et de l'Environnement - ENSE3), Grenoble, France, in 2015. Currently, he is pursuing the Ph.D. degree at the Department of Electrical Energy and Computer Architectures, Katholieke Universiteit Leuven (KU Leuven), Leuven, Belgium. His research interests are power system control and monitoring, optimization of distribution systems and micro-grids.

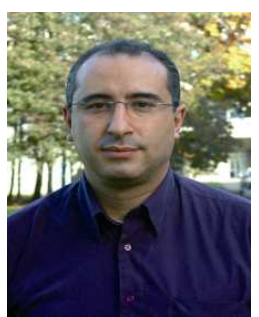

Nouredine Hadjsaid (SM'05) received his Diplôme d'Etudes Approfondies (DEA) and Doctorat de l'INPG degrees from the Institut National Polytechnique de Grenoble (INPG) in 1988 and 1992. From 1988 to 1993 , he served as a research and teaching assistant at the Ecole Nationale Supérieure d'Ingénieurs Electriciens de Grenoble (ENSIEG) and at the Laboratory d'Electrotechnique de Grenoble (LEG). He is now a full time professor at Grenoble INP at the Ecole d'Ingénieurs pour l'Energie, l'Eau et l'Environnement de Grenoble (ENSE3) in the Grenoble Electrical Engineering laboratory (G2Elab). His research interests are power system operation and security. Since 1992, he has been involved as a scientific director for more than 20 industrial and European projects in the power energy sector and critical infrastructures. These projects concern in particular power system security, new technologies to enhance power system control and monitoring, optimization of distribution systems, distributed generation, ancillary services and planning under deregulation, Information and Communication for Energy for example.

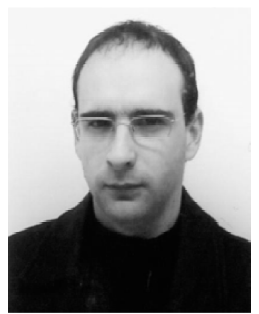

Raphael Caire (M'04 SM'14) received his Diplôme d'Etudes Approfondies (DEA) and Doctorat degrees from the Institut National Polytechnique de Grenoble (INPG) in 2000 and 2004. He had been working in Power Electronic field, in USA at the Center of Power Electronic System (CPES) in 2000 and within several EDF research centers in Germany and in France from 2004 to 2006. He is now associate professor at Grenoble Institute of Technology (Grenoble-INP) at the Ecole Nationale Supérieure de l'Energie, de l'Eau et de l'Environnement (ENSE3) in the Grenoble Electrical Engineering laboratory (G2Elab). His research is centered on the impacts, production and control of dispersed generation on distribution system and critical infrastructures.

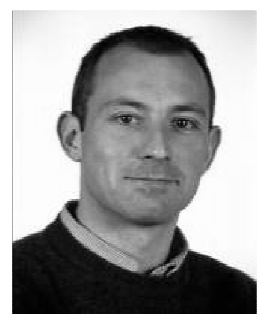

Bertrand Raison (M’03) was born in Béthune, France in 1972. He received his M.S and Ph.D. degrees in electrical engineering from the INPG, France, in 1996 and 2000. He joined for one year the Faculté Polytechnique de Mons, Belgium where he studied the effects of dispersed generation on network stability. He has joined since 2001 for Grenoble (INPG) as associate professor and since 2011 as professor in Université Joseph Fourier the "Laboratoire d'Electrotechnique". His general research interests are fault detection and localization in electrical systems.

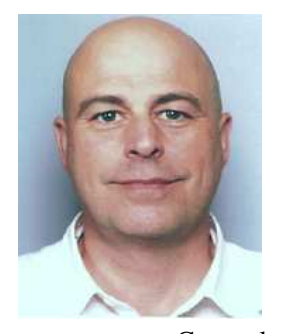

Eric Martin received his Diplôme d'Etudes Approfondies (DEA) from the Institut National Polytechnique de Grenoble (INPG) in 1985 . He worked in France for Merlin Gerin from 1985 to 1992 then for Schneider Electric until now. In 1985 he contributed in the development of the first digital relay of the company and in 1997 for the first neuronal network differential transformer protection. Since 1985 he took different positions in the protection relay activity mainly in $R \& D$ and project management. Currently, he manages the certification and validation of protection relays of Schneider Electric. His general research interests are protection algorithm for Industry and Utilities and power system control and monitoring. 